\title{
Research on vibration Control of traction elevator
}

\author{
Xiaomei Jiang ${ }^{1, a}$, Yannian Rui ${ }^{2, b}$ \\ ${ }^{1}$ School of Mechanical Engineering, Changshu Institute of TechnologyChangshu, 215500, China \\ ${ }^{2}$ College of Mechatronics Engineering, Soochow University, Suzhou, 215021, China \\ ajszjjxm@hotmail.com, ${ }^{b}$ ryn@ @suda.edu.cn
}

Keywords: elevator; vibration analysis; dynamic modeling simulation; adaptive control

\begin{abstract}
Elevator is a necessity in modern people's life; its vibration is the elevator's important evaluation index of comfort. Most of the smaller vibration amplitude of the elevator will not pose a threat to the health of passengers and personal security, but once the elevator vibration reaches a certain value, will bring the passenger discomfort. So to strengthen vibration analysis and control is an important problem, the key vibration control of elevator is to focus on the elevator vibration in human sensitive frequency. Elevator is a complex nonlinear coupling vibration system with multiple degree of freedom, the traditional control method is often difficult to meet the needs of its vibration control, the dynamic model of a high-speed traction-elevator is built up considered time-varying characteristics like the quality of wire rope, stiffness, damping, the dynamic characteristics of the system are analyzed and the vibration control techniques are studied. By acceleration response simulation under the given speed curve, the presented method has relatively adaptive ability to fit vibration environment.
\end{abstract}

\section{Introduction}

The elevator is a necessity in modern people's life; its vibration is the elevator's important evaluation index of comfort. Most of the smaller vibration amplitude of the elevator will not pose a threat to the health of passengers and personal security, but once the elevator vibration reaches a certain value, will bring the passenger discomfort[1]. So to strengthen vibration analysis and control also to reduce vibration can improve the quality of the elevator.

From research about the vibration of elevator home and abroad, the reasons caused the vibration of elevator system include two categories: one is mechanical vibration, namely by the traction machine, weighing device, suspension, car caused by poor design, bad manufacture and installation quality; another kind is the electrical vibration, namely the performance of the motor, drive and control system and inappropriate matching[2].. The key vibration control of elevator is to focus on the elevator vibration in human sensitive frequency, in order to improve the comfort[3].

Elevator is a complex nonlinear vibration system with multiple degrees of freedom, the traditional control method is often difficult to meet the needs of its vibration control, and neural network control shows superior performance in nonlinear control process of complex, big lag, inaccurate mathematical model, implements the control based on the knowledge (rules) and even semantic description and provides a relatively easy design method for the nonlinear controller, especially when the control object or process with uncertainties and difficult to use conventional linear control theory, it is more effective[4,5]. A neural network adaptive vibration control method was adopted for the elevator. In this thesis, high-speed traction elevator vertical vibration system model is established, based on the neural network adaptive active control technology, using MATLAB programming for vibration control simulation, and then go further into actual elevator vibration control and effect.

\section{Vertical vibration dynamics modeling}

Different elevator system has certain difference, even for the same traction type elevator, due to different transmission ratio; mechanical system also has the certain difference. General traction elevator mechanical drive system is mainly composed of traction machine system, traction wheel, 
guide wheel, tension wheel, capsules, balance counterweight, traction rope, tension rope, etc. Due to the different transmission ratio of traction system, the simplified model will have the difference in dynamic analysis; the model built also has the certain difference.

Elevator is a variable parameter system, in running process the length of wire rope and elevator load change, so to establish a time-varying continuous model of elevator is very difficult, but can take the elevator operation process discretization in time domain, assuming every time interval the elevator parameters are the same, and in every interval elevator system can be regarded as a multiple degree of freedom vibration system, its dynamic equation can be expressed as common form of multiple DOF centralized mass system dynamics equation[3]:

$$
M \cdot \ddot{x}+C \cdot \dot{x}+K \cdot x=q \cdot
$$

$M, C$ and $K$ respectively is the system quality matrix, the damping matrix and the stiffness matrix; $x, q$ respectively is movement displacement vector of each DOF and force load vector. Take 2:1 gearless traction elevator as an example, driven by permanent magnet synchronous motor, the rpm of the motor equal to the speed of the traction sheave.

According to the mechanical structure characteristics and vertical vibration mechanism of elevator, without considering guide shoe system (friction force) under the condition of vertical vibration, the vertical vibration dynamics system model is established. In this model, all of the vibration displacement takes linear displacement upwardly as positive, angular displacement counter clockwise as positive. According to the Lagrange equation, establishes the system dynamic equation.

$$
\frac{d}{d t}\left(\frac{\partial T}{\partial \dot{x}_{i}}\right)-\frac{\partial T}{\partial x_{i}}+\frac{\partial D}{\partial \dot{x}_{i}}+\frac{\partial U}{\partial x_{i}}=q_{i}
$$

Total kinetic energy $\mathrm{T}$ is:

$$
\begin{aligned}
& T=\frac{1}{2} m_{0} \dot{x}_{0}^{2}+\frac{1}{2} m_{1} \dot{x}_{1}^{2}+\frac{1}{2} m_{2} x \dot{x}_{2}^{2}+\frac{1}{2} m_{3} \dot{x}_{3}^{2}+\frac{1}{2} m_{4} \dot{x}_{4}^{2}+\frac{1}{2} m_{5} \dot{x}_{5}^{2}+\frac{1}{2} m_{6} \dot{x}_{6}^{2}+\frac{1}{2} m_{7} \dot{x}_{7}^{2}+\frac{1}{2} m_{r 11} \dot{x}_{r 11}^{2}+\frac{1}{2} m_{r 12} \dot{x}_{r 12}^{2}+ \\
& \frac{1}{2} m_{r 21} \dot{x}_{r 21}^{2}+\frac{1}{2} m_{r 22} \dot{x}_{r 22}^{2}+\frac{1}{2} m_{c 1} \dot{x}_{c 1}^{2}+\frac{1}{2} m_{c 2} \dot{x}_{c 2}^{2}+\frac{1}{2} I_{1} \dot{\varphi}_{1}+\frac{1}{2} I_{2} \dot{\varphi}_{2}+\frac{1}{2} I_{3} \dot{\varphi}_{3}+\frac{1}{2} I_{4} \dot{\varphi}_{4}
\end{aligned}
$$

Total potential energy $\mathrm{V}$ is:

$$
\begin{aligned}
& V=\frac{1}{2} k_{p}\left(x_{2}-x_{1}\right)^{2}+\frac{1}{2} k_{3}\left(x_{6}-x_{2}\right)^{2}+\frac{1}{2} k_{r 1}\left(x_{6}-x_{r 11}+R_{3} \varphi_{3}\right)^{2}+\frac{1}{2} k_{r 1}^{*} x_{r 11}^{2}+\frac{1}{2} k_{r 1}\left(x_{r 12}-x_{6}+R_{3} \varphi_{3}\right)^{2}+\frac{1}{2} k_{r 1}\left(x_{3}-x_{r 12}+R_{1} \varphi_{1}\right)^{2}+ \\
& \frac{1}{2} k_{r 2}\left(x_{r 22}-x_{3}+R_{1} \varphi_{1}\right)+\frac{1}{2} k_{r 2}\left(x_{7}-x_{r 22}+R_{4} \varphi_{4}\right)^{2}+\frac{1}{2} k_{r 2}\left(x_{r 21}-x_{7}+R_{4} \varphi_{4}\right)^{2}+\frac{1}{2} k_{r 2}^{*} x_{r 21}^{2}+\frac{1}{2} k_{4}\left(x_{4}-x_{7}\right)^{2}+\frac{1}{2} k_{c 2}\left(x_{c 2}-x_{4}\right)^{2} \\
& +\frac{1}{2} k_{c 2}\left(x_{5}-x_{c 2}+R_{2} \varphi_{2}\right)^{2}+\frac{1}{2} k_{c 1}\left(x_{c 1}-x_{5}-R_{2} \varphi_{2}\right)^{2}+\frac{1}{2} k_{c 1}\left(x_{2}-x_{c 1}\right)^{2}+\frac{1}{2} k_{1} \dot{x}_{3}^{2}+\frac{1}{2} k_{2} x_{5}^{2}+\frac{1}{2} k_{T} \varphi_{1}^{2}+\frac{1}{2} k_{1}\left(x_{3}-x_{0}\right)^{2}+\frac{1}{2} k_{0} x_{0}^{2}
\end{aligned}
$$

Total dissipation energy $\mathrm{D}$ is:

$$
\begin{aligned}
& D=\frac{1}{2} c_{p}\left(\dot{x}_{2}-\dot{x}_{1}\right)^{2}+\frac{1}{2} c_{3}\left(\dot{x}_{6}-\dot{x}_{2}\right)^{2}+\frac{1}{2} c_{r 1}\left(\dot{x}_{6}-\dot{x}_{r 11}+R_{3} \dot{\varphi}_{3}\right)^{2}+\frac{1}{2} c_{r 1}^{*} \dot{x}_{r 11}^{2}+\frac{1}{2} c_{r 1}\left(\dot{x}_{r 12}-\dot{x}_{6}+R_{3} \dot{\varphi}_{3}\right)^{2}+\frac{1}{2} c_{r 1}\left(\dot{x}_{3}-\dot{x}_{r 12}+R_{1} \dot{\varphi}_{1}\right)^{2}+ \\
& \frac{1}{2} c_{r 2}\left(\dot{x}_{r 22}-\dot{x}_{3}+R_{1} \dot{\varphi}_{1}\right)+\frac{1}{2} c_{r 2}\left(\dot{x}_{7}-\dot{x}_{r 22}+R_{4} \dot{\varphi}_{4}\right)^{2}+\frac{1}{2} c_{r 2}\left(\dot{x}_{r 21}-\dot{x}_{7}+R_{4} \dot{\varphi}_{4}\right)^{2}+\frac{1}{2} c_{r 2}^{*} \dot{x}_{r 21}^{2}+\frac{1}{2} c_{4}\left(\dot{x}_{4}-\dot{x}_{7}\right)^{2}+\frac{1}{2} c_{c 2}\left(\dot{x}_{c 2}-\dot{x}_{4}\right)^{2}+ \\
& \frac{1}{2} c_{c 2}\left(\dot{x}_{5}-\dot{x}_{c 2}+R_{2} \dot{\varphi}_{2}\right)^{2}+\frac{1}{2} c_{c 1}\left(\dot{x}_{c 1}-\dot{x}_{5}-R_{2} \dot{\varphi}_{2}\right)^{2}+\frac{1}{2} c_{c 1}\left(\dot{x}_{2}-\dot{x}_{c 1}\right)^{2}+\frac{1}{2} c_{1} \dot{x}_{3}^{2}+\frac{1}{2} c_{2} \dot{x}_{5}^{2}+\frac{1}{2} c_{T} \dot{\varphi}_{1}^{2}+\frac{1}{2} c_{1}\left(\dot{x}_{3}-\dot{x}_{0}\right)^{2}
\end{aligned}
$$

According to the Lagrange equation, the matrix and vector of the motion equation can be determined. Vertical vibration of elevator can be measured through the two acceleration indicators, vibration acceleration peak -peak value and A95 (it is such an acceleration bandwidth, making 95\% of the peaks and troughs fall within the bandwidth, its unit is $\mathrm{m} / \mathrm{s} 2)[6]$.

Select $x=\left\{x_{0}, x_{1}, x_{2}, x_{3}, x_{4}, x_{5}, x_{6}, x_{7}, x_{\mathrm{r} 11}, x_{\mathrm{r} 12}, x_{\mathrm{r} 21}, x_{\mathrm{r} 22}, x_{\mathrm{c} 1}, x_{\mathrm{c} 2}, \varphi_{1}, \varphi_{2}, \varphi_{3}, \varphi_{4}\right\}^{T}$ as state variables, due to the comfort requirement, choose car vibration acceleration as the control target, take it as the output, and make the objective function minimum. Establish state space equation of the system:

$$
\left\{\begin{array}{l}
X=A X+B U \\
Y=C X+D U
\end{array}\right.
$$




\section{Adaptive neural network controller design}

Adaptive neural network control is developed on the basis of the automatic control, especially suitable for hard to describe in the general physical and chemical laws, or don't have any mathematical model of controlled object (or process). Neural network adaptive controller is based on the simple PID feedback controller, is actually control mode combined neural network control system identification with classic controller. Adaptive vibration control technology has strong robustness for random interference and adaptive ability, reasoning more accords with the actual requirements, the expert controller was adopted which contains strategic knowledge, it allows more complex hierarchical rules, and can determine the application which rules between low-level rules, All the rules described above are called "event-driven rules", processing in the so-called forward link mode; these rules can only be applied when state in the process "matching" with pre-determined conditions [7]. The self-learning ability and adaptive good performance characteristics of artificial neural network are used to implement intelligent vibration control. The system consists of six parts: the expected pattern generator, neural network adaptive controller, proportional feedback controller, feedforward controller, parameter setting and acceleration, velocity, load sensor. The system block diagram is shown in Fig. 1.

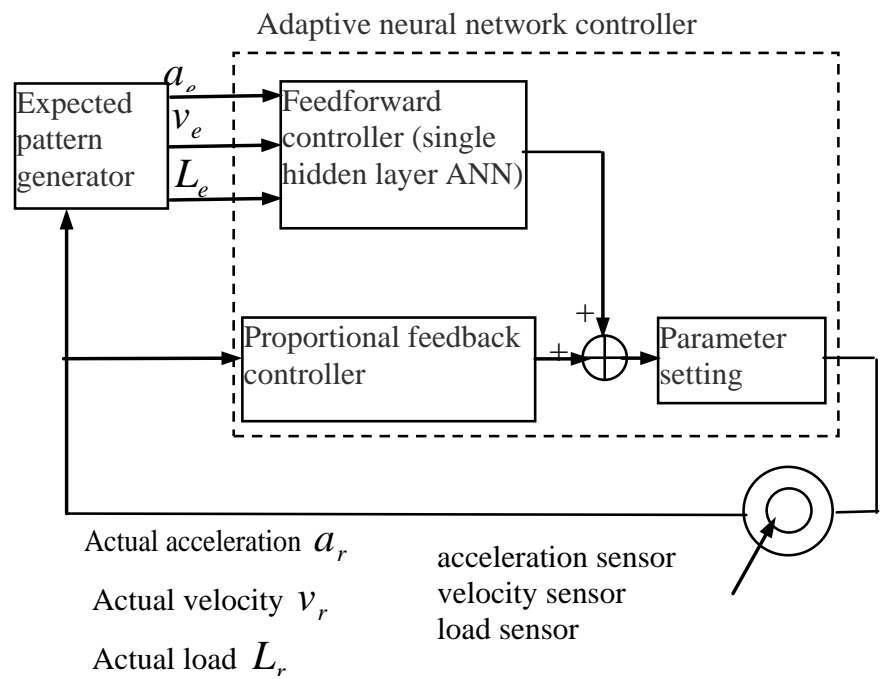

Fig. 1. Intelligent control block diagram

Using neural network adaptive controller to on-line control and monitor the running of the elevator, it can adaptively adjust with the changes of the external excitation signal, information processing results not being stored in a unit, but by practical training and accumulation of repeated experience to make acceleration alarm limits of elevator be close to expectations under different circumstances, which thus play a proactive part.

\section{Simulation result analysis}

In the movement process dynamics parameter of elevator is in the change, in which the car quality at no load and full load changes between. In the different travel altitude, the stiffness of the two sides of wire rope changes along with the car running. In Elevator running process, the excitation is mainly composed of the random factors such as starting, deceleration, braking process. According to the motion elastic dynamic analysis method, use the instantaneous structure hypothesis in the elevator mechanical system; break it into several time-invariant discrete transient systems to solve. Discrete sampling process can be handled by the sampling period $\mathrm{T}$, assuming only in the sampling instant $\mathrm{kT}$, time-varying parameters change. On the basis of the configuration parameters for an elevator, calculate stiffness matrix and mass matrix, damping matrix of the vertical dynamics model of the elevator system. Modal analysis was carried out on the different working conditions. According to the previous analysis, impose sinusoidal excitation respectively on the top of car top sheave, counterweight sheave and traction sheave, selection of full load and no load calculated, with these 
two kinds of working condition in time interval $t=10$ s, by MATLAB program, using the runge-kutta method to solve the system state equation, through simulation, elevator vertical acceleration response curve is acquired as shown in Fig. 2, as can be seen from the diagram, the accelerating running period and slow down running period of elevator, its acceleration amplitude is greater than that of constant speed running period. Its maximum acceleration peak-peak value exceeds $0.6 \mathrm{~m} / \mathrm{s}^{2}$. As can bee seen, in the process of elevator running vibration is relatively intense, in order to improve the vibration behavior of elevator, implementing the preliminary vibration control, using the neural network adaptive control, aiming at working conditions such as different load and different position and the different speed stages of elevator, according to the multiple excitation optimization model, making the elevator parameters optimize, then again applying the obtained optimized result to perform the simulation on the elevator running process, the improved acceleration response curve is acquired as shown in Fig. 3, as can be seen from the diagram, its maximum acceleration peak-peak value is less than $0.5 \mathrm{~m} / \mathrm{s}^{2}$, A 95 value also declines relative to the former.

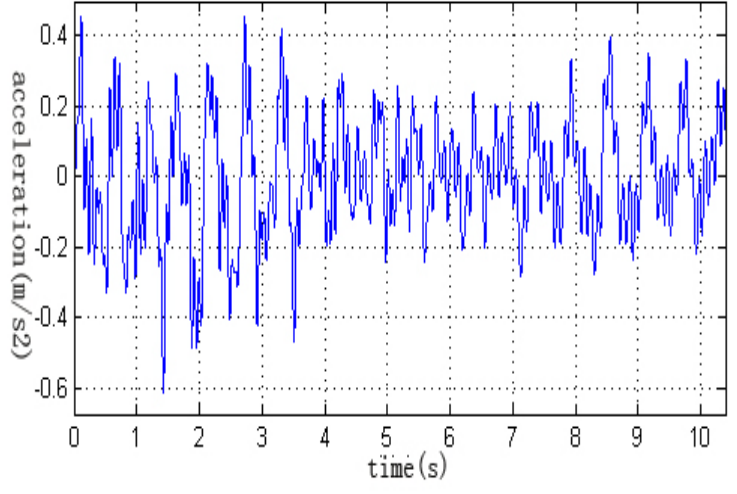

Fig. 2 Acceleration response in running

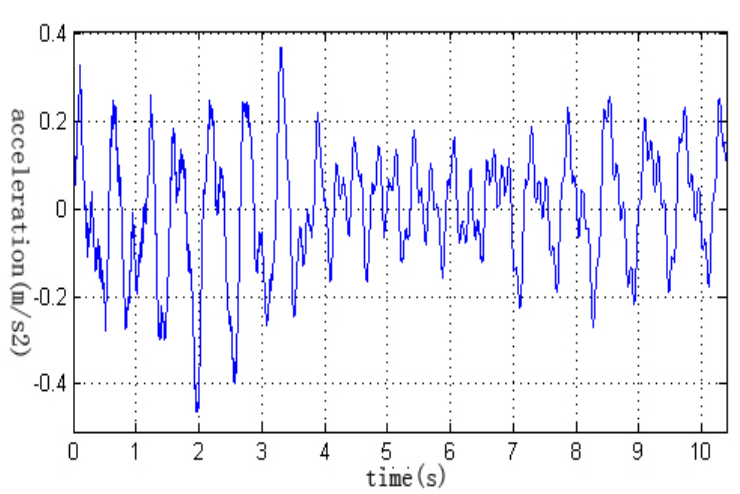

Fig. 3 Improved acceleration response in running

\section{Summary}

Through this research, we can see that the use of neural network adaptive controller can moderately meet the requirement of vibration control. This has great significance for the rapid development of intelligent building industry, at the same time this was also an important aspect of modern intellectual development.

\section{References}

[1] ISO 8041: 2005 Human response to vibration - Measuring instrumentation (2005).

[2] M.Z. Ren, Analysis and control and the calculation methods of mechanical vibration, Beijing: mechanical industry press (2011).

[3] Y.H. Cheng, The Application of Frequency Weight Filtering in Elevator Vibration Measurement, Automation \& Information Engineering, 34 (2013) 33-36.

[4] J.K. Liu, The advanced PID control and MATLAB simulation, Beijing: electronic industry press, (2006).

[5] S. N. Xu and Z. D. Zhou, neural network adaptive control algorithm modified by PSO, China Mechanical Engineering, 23 (2012) 2732-2738.

[6] W.H. Bai, "Study on active vibration control of traction elevator," Practical Electronics, (2012) 110-112.

[7] L.M. Zhang, Model and its Application of Artificial Neural Network, Shanghai: Fudan University Press (2009). 